\title{
DÜBLIN
}

Technological University Dublin

ARROW@TU Dublin

2009-09-01

\section{An Experimental Study of the Impact of Using Multi-Radio in WLAN Mesh Networks}

Chenzhe Zhang

Technological University Dublin, chenzhe.zhang@tudublin.ie

Karol Kowalik

Technological University Dublin, Karol.kowalik@tudublin.ie

Mark Davis

Technological University Dublin, mark.davis@tudublin.ie

Follow this and additional works at: https://arrow.tudublin.ie/commcon

Part of the Systems and Communications Commons

\section{Recommended Citation}

Zhang,C., Kowalik,K. \& Davis, M. (2009) An Experimental Study of the Impact of Using Multi-Radio in WLAN Mesh Networks.5th International Conference on Wireless Communications, Networking and Mobile Computing (WiCom '09), Beijing, China. 24-26 September, 2009.

This Conference Paper is brought to you for free and open access by the Communications Network Research Institute at ARROW@TU Dublin. It has been accepted for inclusion in Conference papers by an authorized administrator of ARROW@TU Dublin. For more information, please contact arrow.admin@tudublin.ie, aisling.coyne@tudublin.ie,gerard.connolly@tudublin.ie. Funder: Science Foundation Ireland

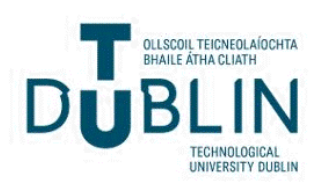




\title{
An Experimental Study of the Impact of Using Multi-Radio in WLAN Mesh Networks
}

\author{
Chenzhe Zhang, Karol Kowalik, Mark Davis \\ Communications Network Research Institute, \\ Dublin Institute of Technology \\ Ireland \\ Chenzhe.zhang@cnri.dit.ie, karol.kowalik@cnri.dit.ie, mark.davis@dit.ie
}

\begin{abstract}
Next-generation wireless mobile communications will be driven by converged networks that integrate disparate technologies and services. Wireless Mesh Networks (WMNs) are expected to be one of the next generation of wireless interconnection technologies, providing flexible high bandwidth wireless backhaul over large geographical areas. While single radio mesh nodes operating on a single channel suffer from capacity constraints, equipping mesh routers with multiple radios using multiple non-overlapping channels can significantly alleviate the capacity problem and increase the aggregate bandwidth available to the network. However, it is well known that multi-radio Mesh platforms face many limitations such as interference, radiation leakage, crosstalk and limited computing resource etc. A misperception among researchers is that the so called "crosstalk" is one of the limiting factors to the performance decrease in multi-radio Mesh networks, but through a series of experiments the results shows otherwise. In this paper, we present a unique experimental approach that utilizes antenna cables, splitters, couplers and attenuators etc. to create a controlled wireless environment, and results indicates, despite negligible effect, crosstalk is not a limiting factor, but rather the finite computing resource constrains the aggregated performance of multi-radio Mesh networks, and several other factors have been investigated as well.
\end{abstract}

Keywords: Cabled wireless mesh networks, multi-radio, performance measurement, testbed..

\section{INTRODUCTION}

There is an increasing interest in using Wireless Mesh Networks (WMNs) as broadband backbone networks to provide ubiquitous network connectivity in enterprises, campuses, and in metropolitan areas. WMNs is a fully wireless network that employs multi-hop ad hoc networking techniques to forward traffic to/from the Internet. Unlike the mobile ad hoc network (MANET), a mesh network uses dedicated nodes (called mesh routers) to build a wireless backbone to provide multi-hop connectivity between nomadic users and the Internet gateways [1]. WMNs can provide significant advantages in deploying cost efficient, highly flexible, and reconfigurable backhaul connectivity over large areas. As highlighted in [2], WMNs have emerged as a promising candidate for extending the coverage of $\mathrm{Wi}-\mathrm{Fi}$ islands and providing flexible high-bandwidth wireless backhaul for converged networks.

The increasing deployment of wireless mesh technologies and infrastructure is leading to an increased usage of applications such as IP telephony, video streaming, public surveillance etc., and in turn these applications often demand much more capacity of the network. But the current state-ofthe-art mesh networks, which use off-the-shelf 802.11-based network cards, are typically configured to operate on a single channel using a single radio. This configuration adversely affects the capacity of the WMNs due to many factors as identified in [3], so as to reduce the channel contention and increase the capacity of mesh network, it's helpful to equip the WMN nodes with multi-radio devices. Therefore the shift from single radio to multi-radio WMNs is an inevitable trend.

But when wireless mesh routers are equipped with one or more radio interfaces, the capacity of the mesh network is highly affected. This has a significant impact on the overall performance of the system, thus generating extensive research in order to tackle the specific challenges of the WMN. (See [3] for a survey).

\section{BACKGROUND AND RELATED WORK}

Equipping each node with multiple radios is emerging as a promising approach to improving the capacity of WMNs. First, the IEEE $802.11 \mathrm{~b} / \mathrm{g}$ and IEEE 802.11a standards provide 3 and 12 non-overlapping (frequency) channels, respectively, which can be used simultaneously within a neighborhood (by assigning non-overlapping channels to radios). This then leads to efficient spectrum utilization and increases the actual bandwidth available to the network. Second, the availability of cheap off-the-shelf commodity hardware also makes multi-radio solutions economically attractive. Finally, the spatial-temporal diversity of radios operating on different frequencies with different sensing-tohearing ranges, bandwidth, and fading characteristics can be leveraged to improve the capacity of the network.

Although multi-radio mesh nodes have the potential to significantly improve the performance of mesh networks, it is much more complex than single-radio WMNs, it faces many constrains and challenges such as channel selection, degradation of overall throughput when using multiple NICs in a node, etc. The ideal $\mathrm{N}$ fold performance improvement is simply not possible to achieve in a real network. Extensive research has been done in the area regarding the performance degradation, and the evaluation has typically been addressed through simulations. However, the research community 
recently started experimentally evaluating mesh routers [4], [5], [6], We should note at this point that the impact of multiple cards has been briefly described in [4] where it was reported that no two $802.11 \mathrm{a}$ and $802.11 \mathrm{~g}$ cards could coexist in the same box without significant performance impact. In addition, in [5] the authors mention that their experiments revealed that Netgear cards require a separation of 6 inches, while Cisco cards appeared to generate interference in the vertical plane, and a vertical separation of 3 inches was necessary for no interference. Furthermore, in [6] the authors suggested that simultaneous activation of multiple radios inside the same node leads to degradation in performance due to: i) board crosstalk, ii) radiation leakage, and iii) inadequate separation between the several antennas. Through experimental study with a focus on examining the existence of crosstalk, it is shown that such crosstalk does not appear to be a limiting factor to the network performance in a controlled wireless environment.

The rest of the paper is organized as follows. Section III briefly introduces the experimental testbed and the set of tools used in the experiments, it also describes the experimental approach and evaluation metrics. Section IV presents the experiment results and performance analysis. Finally Section $\mathrm{V}$ discusses our observations and concludes the paper.

\section{EXPERIMENTAL IMPLEMENTATION}

In this section we describe our controlled wireless network setup and configuration, as well as the set of experiments that were performed and the evaluation metrics used.

\section{A. Experimental testbed}

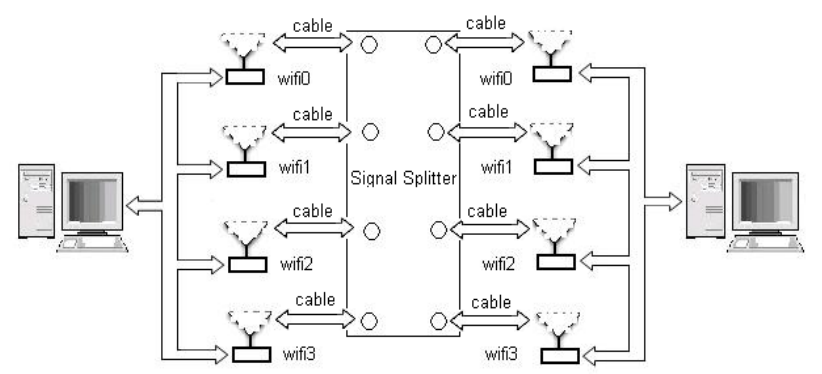

Figure1: Experimental test bed used in this experiment

The experimental testbed comprises 2 PCs that have up to 4 WLAN NICs in each. These 8 NICs have been configured to operate in the Adhoc (i.e. infrastructureless) mode to form 4 pairs operating on orthogonal channels. All NICs are then interconnected by couplers and attenuators through a splitter as shown in Figure 1, this testbed aims to exclude unpredictable external influence. All NICs operate in the 802.11a mode with the antenna diversity feature disabled and a fixed transmission rate of $54 \mathrm{Mbps}$ on each interface.

\section{B. Experimental approach}

1) Experiments at the $T X$ node: Initially, a baseline performance is established by measuring the maximum achievable throughput between a single $\mathrm{Tx} / \mathrm{Rx}$ pair (i.e. involving just 2 NICs). The other 6 NICs are disabled.
Measurements are done by using Iperf [7] toolsets. Having established a stable and reproducible baseline, the impact of multiple wireless interfaces within a node is studied. The intention is to determine if there is any negative impact on throughput performance as a result of cross-talk between the wireless interfaces. During this study, a single receiving node with a single interface is used and the number of transmitting interfaces in the transmitting node is increased until all four PCI slots are occupied. Each time an additional wireless interface card is inserted, the card is set to the monitor (passive) mode and tuned to an orthogonal channel. The maximum achievable throughput is measured as described in step 1 above. The CPU usage is also noted. Step 1 and 2 are then repeated with a different tool (i.e. DITG) [8] to generate a saturated network condition, where the additional cards are set to the AP mode. The throughput results for the saturated network are recorded.

2) Experiments at the $R X$ node: Similar procedures are applied at the RX node, but here different operation modes of the NICs are used while the saturated throughput is recorded. This is to examine the impact of the various operation modes on the network performance. Also the TX transmitting power is varied to study its impact on throughput. During each test, link quality is measured and recorded using the Madwifi driver. The D-ITG tool sets are used to generate a saturated network condition and to record throughput for each scenario.

In both section 1) and 2), tests were carried out for the duration of 60 seconds each and were repeated 3 times to take the average value.

\section{RESULTS AND ANALYSIS}

In this section we present the results and evaluation from the experimental work, this section is divided into two sections corresponding to the 2 sets of experimental approach as described in the previous section. i.e. experiments at both the RX and TX node.

\section{A. Experiments at the TX node}

1) Performance measurement under the non-saturation condition at the TX node:

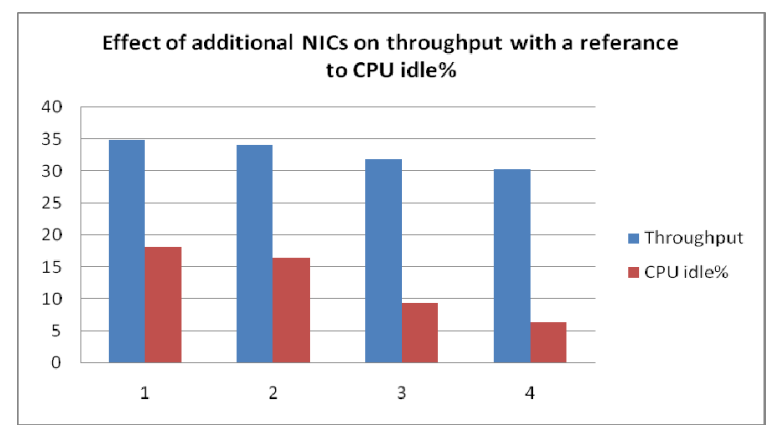

Figure 2: Effect of additional NICs on throughput under non-saturation with a packet size of 1470 bytes. 
Figure 2 indicate that when the network is not saturated and provided that there is sufficient CPU processing power, the capacity of a network decreases as a function of the number of radios. As expected the throughput increases when using larger packet sizes in traffic owing to more efficient use of the transmission medium. It should be emphasized that each additional NIC card has been placed in a monitor (passive) state, and therefore each additional NIC imposes an additional load on the CPU as every frame on the medium is monitored by these additional NICs and hence requires servicing by the CPU. This ultimately leads to a decrease in the throughput performance. This is confirmed by the results in section 2) below.

2) Performance measurement under the saturation condition at the TX node:

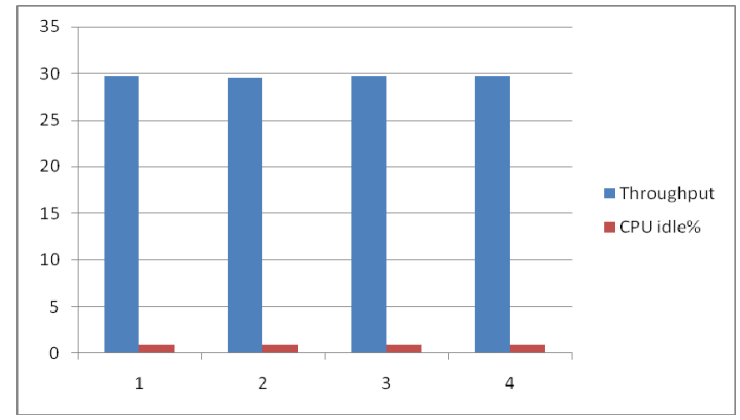

Figure 3: Throughput statistics under saturation condition with a reference to CPUidle\%@1470 bytes.

During this phase of the experiment, the D-ITG tool sets are used to generate large amounts of UDP traffic in order to fully saturate the network and the CPU. The packets are 1400 bytes in size and are created at the rate of 5000 every single second. From the data analysis carried out after the experiments, it is observed that CPU idle $\%<5 \%$ throughout the tests. Furthermore, Figure 3 shows that the throughput does not vary even when additional NICs are inserted. This result fully confirms the observation made in Section A that the decrease in throughput is primarily due to the finite processing power, i.e. crosstalk does not appear to be a limiting in determining the maximum achievable throughput.

Data analysis also show the throughput comparison when the additional NICs are set to the Monitor mode and the AP mode under saturation conditions. There is a slight increase in throughput when using the AP mode which is due to the fact that AP mode does not require that much CPU resources when compared to the Monitor mode.

\section{B. Experiments at the $R X$ node}

This section presents the results that suggest the impact of different modes, transmitting power, link quality on the saturated throughput.

\begin{tabular}{|c|c|c|c|c|c|}
\hline $\begin{array}{c}\text { Signal } \\
\text { Level } \\
(\mathrm{dBm})\end{array}$ & $\begin{array}{c}\text { Noise } \\
\text { Level } \\
(\mathrm{dBm})\end{array}$ & $\begin{array}{c}\text { TP } \\
(\mathrm{Mbps})\end{array}$ & $\begin{array}{c}\text { Link } \\
\text { Quality }\end{array}$ & $\begin{array}{c}\text { No.of } \\
\text { active } \\
\text { NICs }\end{array}$ & $\begin{array}{c}\text { Operational } \\
\text { Modes }\end{array}$ \\
\hline-46 & -84 & 29.7 & $40 / 70$ & 1 & adhoc \\
\hline-46 & -84 & 29.5 & $38 / 70$ & 2 & adhoc \\
\hline-51 & -91 & 29.5 & $38 / 70$ & 2 & monitor \\
\hline-38 & -84 & 29.6 & $45 / 70$ & 2 & inactive \\
\hline-50 & -85 & 29.6 & $35 / 70$ & 2 & adhoc+10mw \\
\hline-54 & -84 & 29.4 & $30 / 70$ & 2 & adhoc $+5 \mathrm{mw}$ \\
\hline-64 & -86 & 16.7 & $22 / 70$ & 2 & adhoc+1mw \\
\hline-51 & -91 & 29.4 & $40 / 70$ & 3 & adhoc \\
\hline-51 & -91 & 29.6 & $40 / 70$ & 3 & monitor \\
\hline-48 & -91 & 29.7 & $43 / 70$ & 3 & inactive \\
\hline-56 & -92 & 29.6 & $36 / 70$ & 3 & adhoc+10mw \\
\hline-60 & -92 & 29.5 & $32 / 70$ & 3 & adhoc+5mw \\
\hline-66 & -93 & 15.2 & $23 / 70$ & 3 & adhoc+1mw \\
\hline-55 & -92 & 29.3 & $37 / 70$ & 4 & adhoc \\
\hline-56 & -93 & 29.4 & $36 / 70$ & 4 & monitor \\
\hline-51 & -93 & 29.6 & $42 / 70$ & 4 & inactive \\
\hline-60 & -93 & 29.5 & $33 / 70$ & 4 & adhoc+10mw \\
\hline-65 & -93 & 29.6 & $29 / 70$ & 4 & adhoc+5mw \\
\hline-71 & -93 & 14.8 & $22 / 70$ & 4 & adhoc+1mw \\
\hline
\end{tabular}

Table 1: Results recorded at the RX node under different test scenarios.

- Both the signal level and noise level decreases when the number of active NICs at the RX node increases.

- When the additional NICs are placed in inactive mode, it can be observed that the signal level increases as well as the link quality plus a slight increase in the throughput.

- There is no significant change in the throughput when additional NICs are inserted into the RX node.

- There is no major difference in throughput between various operation modes.

- A significant decrease in throughput can be observed when the TX transmitting power is set to minimal.

- Throughput changes in relation to link quality.

\section{CONCLUSION}

In this paper we have presented an experimental study of the impact of using multiple radios in wireless networks, its key feature is by creating a cabled wireless testbed, we eliminated external factors that could affect the performance. It is then identified that when given a non-saturated network condition, the capacity of a network increases as a function of the number of active radios, and it is not the case when a saturated condition is present. Furthermore, it is observed that board crosstalk does not appear to be a limiting factor to the degradation of network performance when compared with finite computing resource. It is also studied that various operation modes does not generate significant differences in terms of throughput. 


\section{ACKNOWLEDGMENT}

The authors acknowledge the support of Science Foundation Ireland (SFI) under the Research Frontiers Program.

\section{REFERENCES}

[1] R. Bruno, M. Conti, and E. Gregori, "Mesh Networks: Commodity Multi-hop Ad Hoc Networks," IEEE Commun. Mag., Mar. 2005, pp. 123-31.

[2] R. Karrer, A. Sabharwal and E. Knightly, "Enabling Large-scale Wireless Broadband: The Case for TAPs," 2nd Wksp. Hot Topics in Wireless, Nov. 2003.
[3] I. Akyildiz, X. Wang, and W. Wang, "Wireless Mesh Networks: A Survey," Comp. Networks, vol. 47, no. 47, 2005, pp. 445-87.

[4] R.Draves, J.Padhye, and B.Zill, "Routing" in multi-radio, multi-hop wireless mesh networks," in ACM Mobicom, Philadelphia, PA, 2004.

[5] A.Adya, P.Bahl, J.Padhye, A.Wolman, and L.Zhou. "A multi-radio unification protocol for IEEE 802.11 wireless networks," in Broadnets. 2004.

[6] J.Robinson, K.Papagiannaki, C.Diot, X.Guo, and L.Krishnamurthy. "Experimenting With a Multi-Radio Mesh Networking Testbed," in WinMee, 2005.

[7] Iperf toolsets. Available at http://sourceforge.net/projects/iperf

[8] D-ITG toolsets. Available at http://www.grid.unina.it/software/ITG 